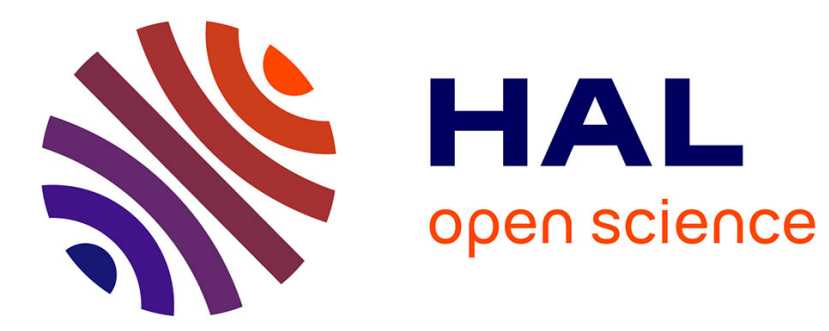

\title{
Des réformes territoriales qui posent bien des questions
}

André Torre, Sébastien Bourdin

\section{To cite this version:}

André Torre, Sébastien Bourdin. Des réformes territoriales qui posent bien des questions. Population et avenir, 2016, 727 (727), pp.14. 10.3917/popav.727.0014 . hal-02002170

HAL Id: hal-02002170

https://hal-normandie-univ.archives-ouvertes.fr/hal-02002170

Submitted on 31 Jan 2019

HAL is a multi-disciplinary open access archive for the deposit and dissemination of scientific research documents, whether they are published or not. The documents may come from teaching and research institutions in France or abroad, or from public or private research centers.
L'archive ouverte pluridisciplinaire $\mathbf{H A L}$, est destinée au dépôt et à la diffusion de documents scientifiques de niveau recherche, publiés ou non, émanant des établissements d'enseignement et de recherche français ou étrangers, des laboratoires publics ou privés. 


\title{
Des réformes territoriales qui posent bien des questions
}

\author{
André Torre ${ }^{1}$ et Sébastien Bourdin ${ }^{2}$
}

Redécouper et fusionner les Régions, redéfinir la place des départements, favoriser le regroupement des communes, créer des métropoles, réduire les dépenses des collectivités territoriales, améliorer la proximité avec les habitants et associer davantage les citoyens aux décisions, voilà les attendus de la loi NOTRe, qui a refondu l'organisation territoriale de la République et défini les fonctions comme la géographie des nouvelles Régions. Cet agenda ambitieux et indigeste, aux objectifs aussi variés que contradictoires, est-il tenable? Quels défis vont devoir affronter les nouveaux responsables de Régions, liés aux objectifs, avantages et risques de la réforme ?

\section{Des objectifs contestables}

L'argument le plus souvent présenté est celui de la simplification et de la clarification du " millefeuille " territorial, facteur de paralysie par sa complexité et ses couches multiples (Communes, intercommunalités, départements, métropoles, Régions...). Le nouveau partage des tâches vise à simplifier le quotidien des habitants et des entreprises dans leurs démarches afin d'améliorer l'efficacité des dispositifs d'aide. Dorénavant, la planification et l'action économique - comme les aides directes aux entreprises - seront réservées aux Régions et l'action sociale et les solidarités aux départements, alors que les communes et leurs regroupements seront en charge de l'urbanisme et de l'organisation des services publics du quotidien. Mais cette simplification est un leurre. Au lieu des ciseaux et de la colle pour redessiner la carte idéale des Régions, il aurait fallu un fouet de pâtissier pour élaborer un nouveau millefeuille avec moins de couches, ou repenser sa forme. Réduire le nombre de Régions ne revient pas automatiquement à simplifier l'organisation administrative territoriale française, et l'augmentation de taille ne fera qu'accentuer la nécessité d'un échelon de proximité. Si l'avenir des départements reste en pointillé, l'intercommunalité pourrait jouer ce rôle, justifiant la montée en puissance des regroupements de communes vers le seuil d'au moins 15000 habitants, suite aux injonctions de l'Etat.

L'objectif de rationalisation de la dépense publique par le passage de 22 à 13 Régions métropolitaines (cf. carte 1) est tout aussi discutable. Pour 2014, ces dernières représentent seulement $12,5 \%$ des dépenses réelles des collectivités territoriales (sur un total de 229,6 milliards ${ }^{3}$ ), ce qui n'est pas excessif au regard de leurs compétences de plus en plus larges. Ces économies ne devraient-elle pas être réalisées sur le fonctionnement de l'Etat central (dont les charges de fonctionnements - hors rémunérations des salariés - s'élèvent à 147 milliards d'euros) plutôt que sur les collectivités qui investissent pour leurs territoires?

Le troisième objectif est de réaliser des économies d'échelle par accroissement de la taille des Régions et des intercommunalités. Lors de la présentation du projet de loi le gouvernement avait annoncé des économies de l'ordre de 25, puis 15 milliards d'euros. Devant le scepticisme général, ce

\footnotetext{
${ }^{1}$ Directeur de recherche à l'INRA, Agroparistech, Université Paris-Saclay : torre@agroparistech.fr

2 Enseignant-chercheur à l'Institut du Développement Territorial, Ecole de Management de Normandie : sbourdin@em-normandie.fr

${ }^{3}$ Trois quarts du budget correspondent au fonctionnement et le dernier quart à l'investissement. Il faut noter que les dépenses de personnel représentent $35 \%$ des dépenses totales de fonctionnement des collectivités territoriales en 2014. Elles sont essentiellement portées par les communes (62\%), puis par les départements (21 $\%)$, les intercommunalités (12\%) et les régions (5\%). Source : DGFiP
} 
chiffre - dont on a du mal à percevoir les modalités de calcul - a été revu à la baisse. Il serait finalement de 10 milliards sur dix ans, moyennant un effort global de réduction des dépenses des collectivités territoriales de l'ordre de $5 \%$ à $10 \%$. Or, même si des économies sont réalisées dans les postes de fonctionnement en supprimant les doublons, en réduisant le nombre d'élus et en mutualisant les services, l'essentiel des dépenses - par exemple les TER ou les lycées - ne sera pas divisé par deux. Enfin, la moins grande flexibilité et la perte de proximité résultant de la fusion des Régions peuvent être à l'origine de surcoûts.

Il s'agit enfin de rechercher un accroissement de compétitivité des Régions. L'idée du Big is beautiful est d'inscrire la France dans la compétition mondiale avec des entités plus visibles et plus fortes et des métropoles au poids renforcé. On peut se demander si Aquitaine ou Rhône-Alpes étaient à ce point étriquées qu'elles nécessitaient une fusion avec d'autres Régions limitrophes ? D'autant plus qu'il n'y a pas de lien prouvé entre la taille des Régions et leur dynamisme (Dumont, 2015). La compétitivité ne se décrète pas, elle se construit, avec une stratégie de long terme et un budget adéquat.

\section{Des avantages ambigus}

Le premier avantage concerne la rationalisation de l'action publique et la clarification des compétences, en particulier entre les différentes collectivités territoriales. La réforme conduit à une redistribution limitée mais bien réelle des compétences, en particulier entre Régions et départements, qui ont sauvé leur peau au prix de l'abandon d'une partie de leurs prérogatives. L'opération de simplification est patente et promise à de nouveaux développements dès 2020 pour les départements. L'abandon de la clause de compétence universelle peut également être considéré comme une avancée contribuant à identifier les dévolutions de chacun des niveaux, mettant un frein à l'éparpillement des dépenses et limitant la volonté d'intervention tous azimuts.

Le deuxième acquis est d'avoir accru la légitimité du rôle joué par les Régions, ne serait-ce que par l'ampleur médiatique du débat concernant les frontières géographiques et les regroupements. Cette discussion a contribué à leur reconnaissance définitive comme l'une des grandes organisations de l'Etat, devant les départements ou les communes, et plus personne ne conteste leur prééminence dans l'architecture de la République. On peut également espérer que les Régions vont jouer un rôle plus important au niveau européen, en portant de manière plus efficace les espérances et initiatives provenant des territoires dans la compétition internationale.

Un autre intérêt de la réforme serait d'avoir donné un pouvoir d'initiative aux forces vives de la nation, ou du moins à ses composantes les plus importantes en volumes de population. Le renforcement du rôle et des compétences des métropoles (Brennetot et de Ruffray, 2015), la reconnaissance du caractère fortement urbain de la population française, un rééquilibrage en faveur des zones les plus peuplées, pourraient conduire à de nouvelles dynamiques, ainsi qu'à une représentation plus équilibrée des actifs, en particulier au moment des prises de décision. Cette conception conduit à accorder une attention toute particulière aux 14 métropoles, créées en 2010 (loi MAPTAM), à leur organisation et à leur fonctionnement, ainsi qu'à les doter d'outils de gestions et de financements appropriés. Le maillage des villes correspondrait à celui de la France contemporaine, traduisant un renversement historique de tendance. Alors que les politiques d'aménagement se sont longtemps inquiétées de "Paris et du désert français ", l'urbain deviendrait la seule voie/levier possible de développement. Les aires métropolitaines imposent leurs singularités, signant un relatif déclin des départements et même des communes, alors que Paris est maintenant parfois vu comme une chance. 
Sebastien Bourdin | Regional Science \& Economic Geography

Find out more publications on sebastienbourdin.com

\section{Des risques réels}

Mais, à côté de ces points positifs, apparaissent également un certain nombre de risques que la réforme fait courir aux collectivités et à leurs populations (Torre et Bourdin, 2015).

Le problème le plus évident est lié à la taille des nouvelles régions; certaines sont devenues de véritables mastodontes (comme Aquitaine - Centre - Poitou Charente ou Rhône-Alpes - Auvergne). Cet accroissement de volume va provoquer un éloignement d'une partie des populations des centres de décision. De nombreux élus ou responsables locaux vont se trouver à plus de deux ou trois heures de route de leur capitale régionale, avec une difficulté à se faire entendre et à porter la parole et les intérêts des populations. L'éloignement, couplé au règne des métropoles, pourrait conduire au sentiment d'un nouveau retrait de l'Etat des territoires périphériques ou ruraux, considérés comme laissés à l'abandon.

Mais surtout, il faut s'attendre à un effet négatif des reconfigurations régionales sur l'équité territoriale. La fusion des Régions ne pourra que renforcer la concentration des activités dans les zones les plus productives. Et provoquera une diminution de la qualité, voire un manque, des services de proximité, à moins d'en installer de nouveaux ou de multiplier les antennes publiques locales, ce qui ne va ni dans le sens de l'histoire ni d'une diminution des coûts. On peut légitimement s'inquiéter pour les habitants des zones "frontalières " ou des territoires les plus éloignés des grandes villes ou des métropoles, dans un contexte de diminution des ressources publiques, de rationalisation des équipements et de suppression de nombreux services de proximité (lycées, formation professionnelle, hôpitaux, postes...) ou de lignes ferroviaires. On touche ici aux contradictions d'un Etat régulateur, qui souhaite diminuer les inégalités entre Régions en réduisant leur nombre mais fait courir le risque d'un accroissement des disparités territoriales internes à chaque Région. En témoigne l'exemple de la région Alsace-Champagne-Ardenne-Lorraine qui cumule de nombreuses difficultés internes notamment en termes de développement économique (seuls deux départements sur dix ont un $\mathrm{PIB} /$ hab. supérieur à la moyenne de l'Union européenne !). Il en résulte des disparités interterritoriales qui sont très prégnantes. Alors que certains départements cumulent les difficultés, comme les Ardennes, les Vosges ou la Moselle, d'autres ont des indicateurs socio-économiques au beau fixe, comme la Marne et le Bas-Rhin dont le dynamisme économique est porté par les anciennes capitales régionales. Strasbourg devient incontestablement le centre de gravité de la nouvelle région, au risque de creuser les écarts devenus structurels.

Un autre risque est lié à l'absence de spécialisation. Alors que la politique européenne de spécialisation intelligente à l'Horizon 2020 met l'accent sur le choix d'un nombre limité d'activités ou de technologies par Région et donc d'une différenciation des fonctions et des productions, on peut craindre l'effet inverse en France. Organisées autour de leurs métropoles, les macro-Régions seront tentées de se comporter comme de petits Etats, reproduisant les compétences et les spécialisations internes sans faire de choix réels de développement, au risque d'un émiettement et d'une banalisation (Béhar, 2015). Il pourrait en résulter une perte de compétitivité et d'attractivité, amplifiée par leur déficit d'image de marque. Pour le moment, les fusions débouchent, en application même de la loi, sur des appellations creuses - comme "Grand Est » ou encore " ACAL " pour Alsace-ChampagneArdenne-Lorraine - peu identifiables et qui ne définissent en aucun cas une identité régionale, pour les habitants comme pour les entreprises ou les consommateurs.

Une inquiétude majeure provient des incertitudes sur les liaisons entre collectivités territoriales, et tout particulièrement sur le couple Région/métropoles, d'autant que ces dernières se voient attribuer une plus grande autonomie et des fonctions étendues, voire un rôle moteur. II s'agit des collaborations 
Sebastien Bourdin | Regional Science \& Economic Geography

Find out more publications on sebastienbourdin.com

entre niveaux, mais encore davantage de la capacité à générer ensemble des effets d'entrainement ou de développement et d'initier des dynamiques communes. La suppression de la clause de compétence générale pourrait réduire l'impact de l'action des collectivités, en les cloisonnant à un domaine d'action défini, alors que le développement territorial suppose au contraire du multidimensionnel et des synergies multiples. De plus, cette limite à la capacité d'action des collectivités sera financièrement contrainte par leurs obligations budgétaires.

Enfin, la réforme suscite différentes interrogations, qui touchent au rôle et à la place de l'Etat. Quel est l'avenir des fonctionnaires territoriaux et des services déconcentrés en cas de fusion ou de suppression ? Quels seront les impacts économiques et sociaux de la fermeture, de la réduction ou du transfert des effectifs, en termes de dynamiques de développement ou de foncier par exemple ? Dans un tout autre domaine, va-t-on assister à un processus de recentralisation, au profit de Préfets tout puissants dans leurs Régions, et disposant de moyens d'action sans commune mesure avec ceux des collectivités ? Sans parler des coûts associés à la réforme : la fusion devrait se solder par un surcoût budgétaire d'environ $1 \mathrm{M} \mathrm{d}^{\prime} €$, du fait du déplacement des services, de leur intégration et de l'alignement des grilles salariales des fonctionnaires territoriaux, dont le nombre variera peu, alors que les économies à attendre sont faibles du fait de la bonne gestion régionale, du moins si l'on en croit un rapport de Septembre 2015 de l'agence Standard \& Poor's.

\section{Une chance paradoxale pour les territoires?}

Mais tout de même, on peut se demander si cette réforme mal fichue ne pourrait pas constituer une opportunité inattendue pour certains territoires ? En effet de nombreux exemples attestent de la capacité d'innovation et de créativité des territoires ruraux ou périphériques. Circuits courts, agriculture paysanne ou épiceries solidaires permettent d'identifier la provenance des produits et d'éviter des intermédiaires tout en rapprochant producteurs et consommateurs. C'est aussi vrai des expériences de collaborations comme les levées de financements communs (crowdfunding), le soutien collectif de projets, les prêts entre particuliers, ou l'épargne de proximité... voire la mise en place de monnaies locales. Ou du crowdsourcing, qui rassemble des collectifs innovants au sein de laboratoires d'idées. Développant ses réseaux de coopération locaux, l'économie sociale et solidaire contribue à l'innovation sociale ou sociétale. Entreprises partagées ou collaboratives (Scops), coopératives d'activité et d'emploi, organisations de transport communautaires, mutualisation des soins, crèches parentales, participent de la résilience des territoires par leur capacité à créer des proximités et maintenir des solidarités locales (Torre, 2015).

Ces nouvelles pratiques s'avèrent précieuses dans les régions où reculent les structures économiques et sociales traditionnelles, avec la disparition des services de proximité comme les magasins et épiceries, les bureaux de postes ou les antennes d'hôpitaux, contribuant à la désertification et à l'isolement des personnes. Elles sont à la base d'un fonctionnement économique davantage axé sur les territoires, mais surtout elles permettent de créer et de maintenir un tissu social fort en permettant de colmater les fractures territoriales et de limiter la montée des délaissés ou des relégués des espaces ruraux ou périurbains. Alors, la nouvelle loi serait-elle finalement une chance pour les territoires, en les oubliant et leur laissant la chance de l'expérimentation tranquille, perdus aux frontières de macro-Régions et loin des Pouvoirs publics ? Ce ne serait pas le moindre de paradoxes d'une réforme qui n'en est pourtant pas avare...

\section{Références :}


Sebastien Bourdin | Regional Science \& Economic Geography

Find out more publications on sebastienbourdin.com

Behar D. (2015), Réforme territoriale: la fin d'un cycle ? L'Économie politique, n4, 36-46.

Brennetot A., de Ruffray S. (2015), Une nouvelle carte des régions françaises, Géoconfluences, juillet, en ligne.

Dumont G.F. (2015), Dix questions sur la nouvelle délimitation des régions, In Torre A., Bourdin S. (eds.), Big Bang Territorial. La réforme des régions en débat, Ed. Armand Colin, Paris.

Standard \& Poor's Ratings Services (2015);

https://www.spratings.com/documents/20184/86966/Nouvelles+regions/87ee2cea-0043-4bdc-

8f74-f3b6ed6dbe34

Torre A. (2015), Théorie du développement territorial, Géographie, Économie, Société, 17, 273-288.

Torre A., Bourdin S. (eds.) (2015), Big Bang Territorial. La réforme des régions en débat, Ed. Armand Colin, Paris (e-book). 


\section{Cartes}

\section{Carte 1 : Les régions avant et après la réforme territoriale}

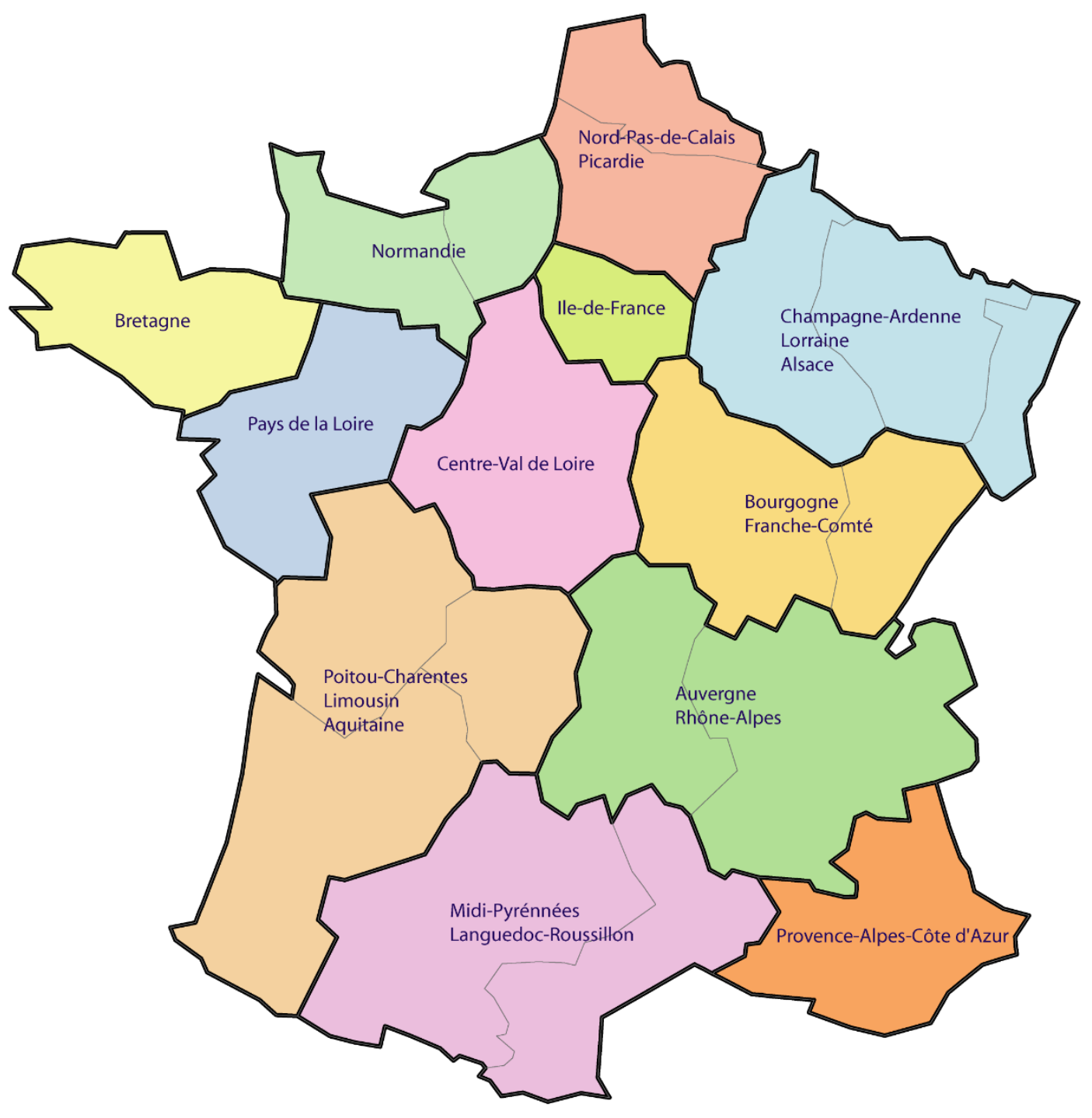


Sebastien Bourdin | Regional Science \& Economic Geography

Find out more publications on sebastienbourdin.com

La Région ACAL : un exemple des inégalités intra régionales

Carte 1 : Part des foyers fiscaux non imposables dans l'ensemble des foyers fiscaux

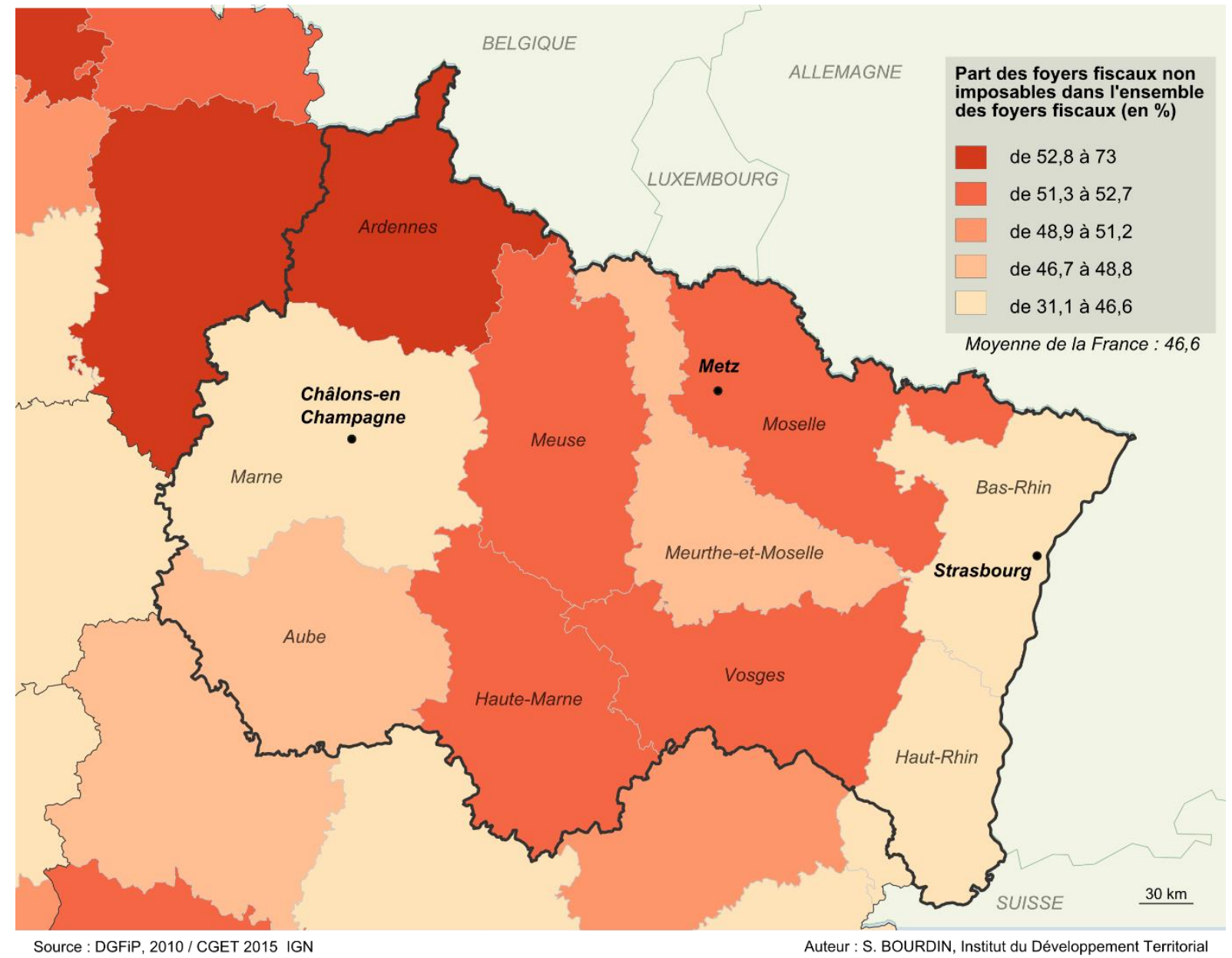

\section{Commentaire}

Le niveau de vie des habitants de l'ex-Champagne-Ardenne et de l'ex-Lorraine est l'un des plus faibles des régions de France métropolitaine. A l'inverse, le niveau de vie des Alsaciens est au deuxième rang derrière l'île-de-France. Les inégalités territoriales en termes d'imposition des ménages révèlent en réalité des inégalités quant à la structure de l'emploi. Alors que l'on observe une surreprésentation des ouvriers et des employés dans la Moselle, la Meuse ou encore les Ardennes, les professions intellectuelles supérieures et les cadres sont davantage concentrés à Strasbourg, Nancy et Châlons-enChampagne. 


\section{Carte 2 : Taux de chômage des 15-24-ans}

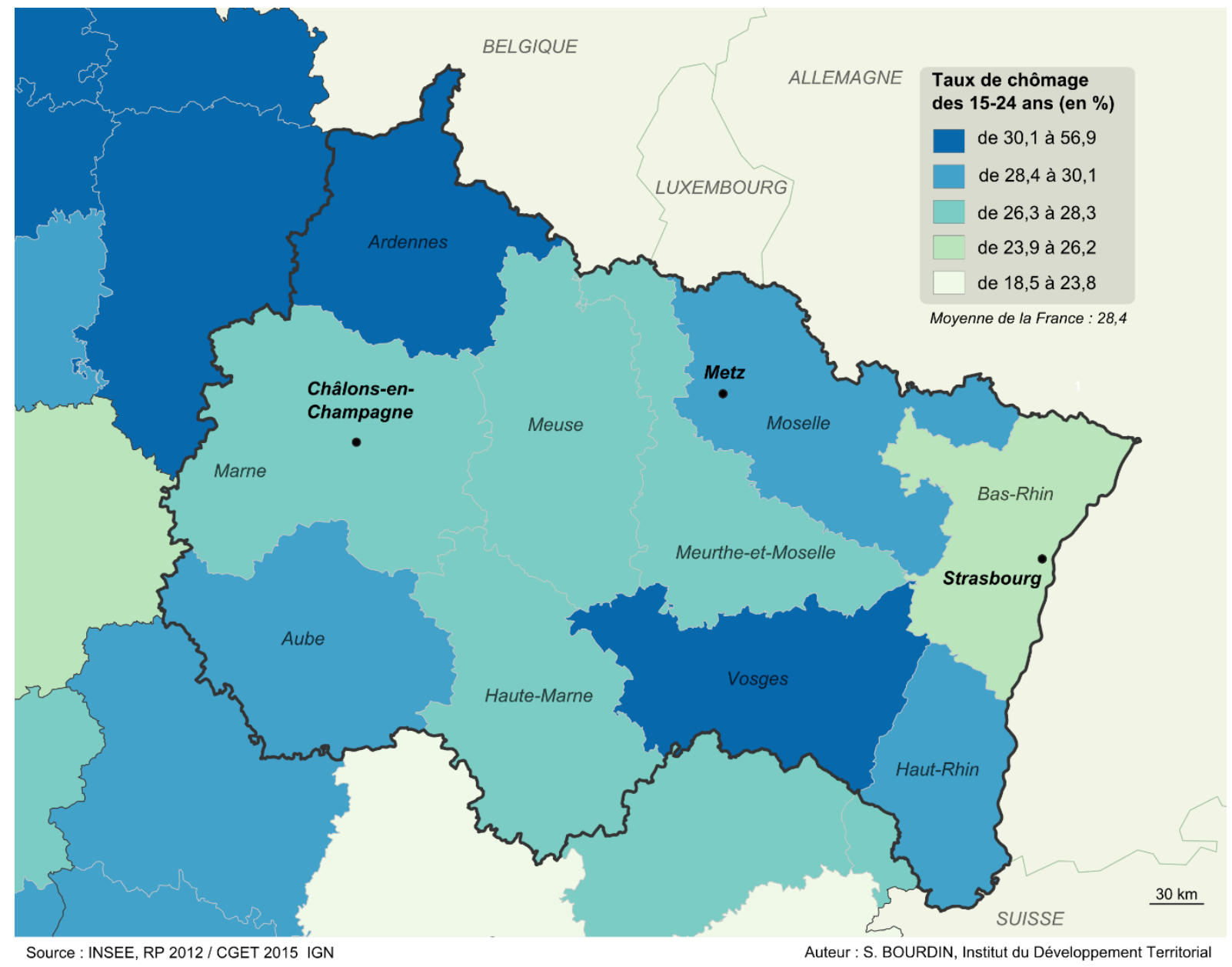

\section{Commentaire}

D'une manière générale, la population augmente moins fortement que la moyenne française et reste jeune. La question de l'emploi des 15-24 ans est au centre des débats pour l'ACAL. Le fait que seuls deux départements enregistrent un taux de chômage des jeunes supérieur à la moyenne nationale $(28,4 \%)$ ne doit pas faire oublier que le travail des frontaliers joue un rôle important dans l'absorption de ce chômage. Pour le département des Ardennes par exemple, la frontière wallonne est moins attractive que la frontière allemande pour la Moselle ou le Bas-Rhin. Par ailleurs, la difficile reconversion industrielle de la région ne facilite pas l'insertion professionnelle de ceux-ci. La part des étudiants dans l'enseignement supérieur (Alsace : 37,5\% ; Lorraine : 40,4\% ; Champagne-Ardenne : 22,1\%) et la répartition des apprentis entre les trois ex-régions (Alsace : 39,4\% ; Lorraine : 39,7\% ; Champagne-Ardenne : 20,9\%) révèlent de profondes inégalités qu'il sera difficile de résoudre. C'est pourtant à cette condition qu'un rééquilibrage territorial du chômage des jeunes pourra se dessiner. 
Sebastien Bourdin | Regional Science \& Economic Geography

Find out more publications on sebastienbourdin.com

\section{Carte 3 : Ecart à la moyenne européenne des PIB départementaux par habitant}

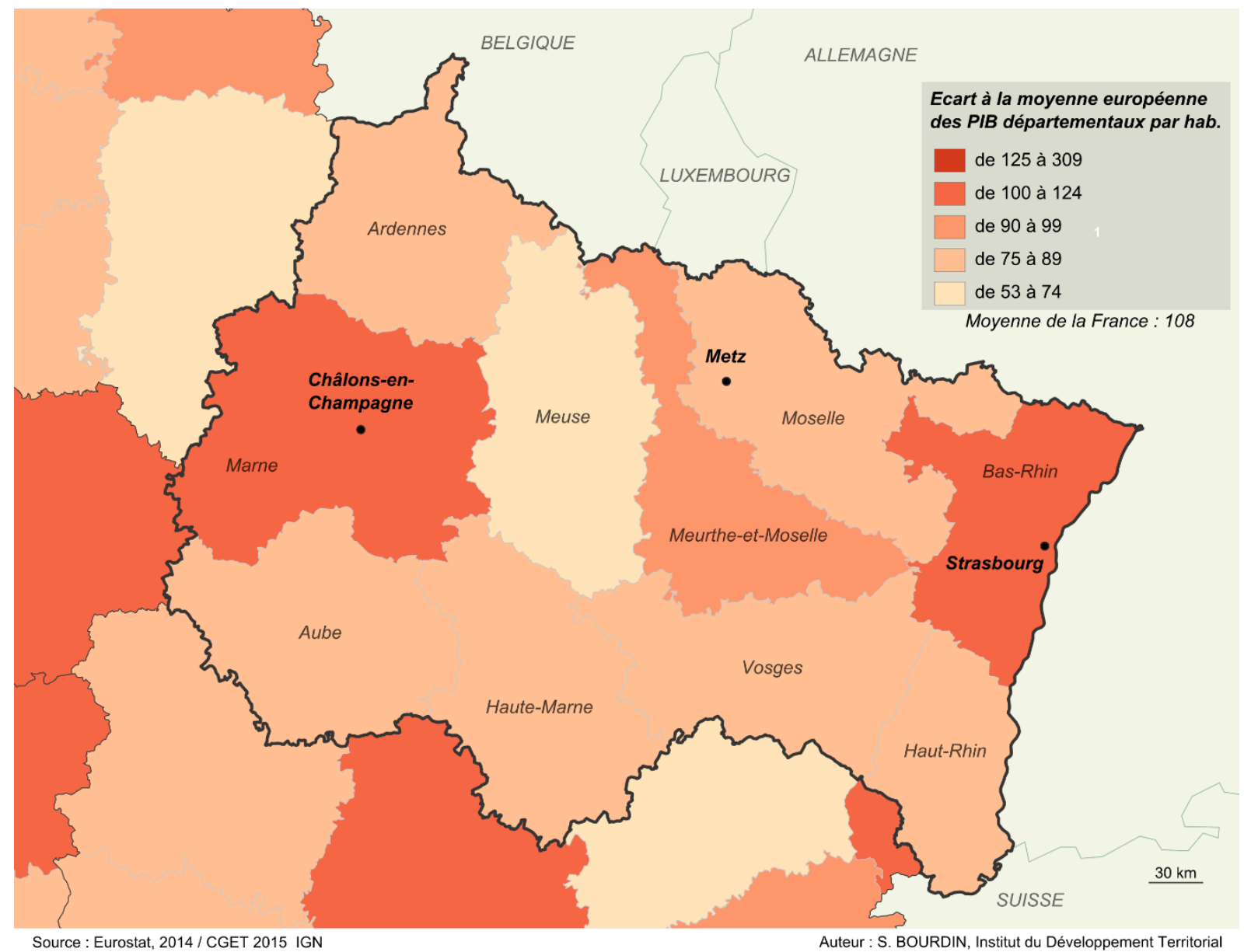

\section{Commentaire}

Excepté pour la Marne et le Bas-Rhin dont les dynamiques économiques sont portées respectivement par Châlons-en-Champagne et Strasbourg, une large partie des départements de l'ACAL sont touchés par une déprise agricole et une faiblesse industrielle. La difficulté aujourd'hui réside dans le fait que I'Alsace s'est orientée vers l'Est avec ses voisins outre-Rhin, tournant ainsi le dos à la Lorraine. Dans ce cadre, la redynamisation économique de la région est suspendue à sa capacité à pouvoir connecter l'ensemble de son territoire à la « dorsale européenne». 